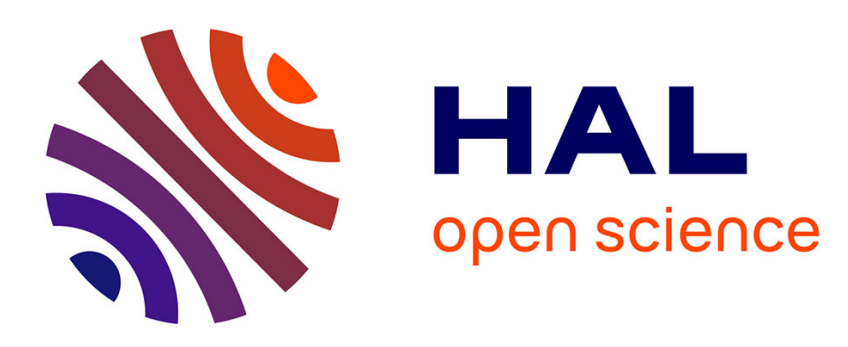

\title{
Large amplitude motion within acetylene-rare gas complexes hosted in helium droplets
}

Marc Briant, Alexandra Viel, Ephriem Mengesha, Marc-André Gaveau,

Benoît Soep, Jean-Michel Mestdagh, Pierre Jamet, Jean-Michel Launay, Lionel Poisson

\section{To cite this version:}

Marc Briant, Alexandra Viel, Ephriem Mengesha, Marc-André Gaveau, Benoît Soep, et al.. Large amplitude motion within acetylene-rare gas complexes hosted in helium droplets. Physical Chemistry Chemical Physics, 2019, 21 (3), pp.1038-1045. 10.1039/C8CP04609C . hal-02443742

\section{HAL Id: hal-02443742 \\ https://hal.science/hal-02443742}

Submitted on 11 May 2020

HAL is a multi-disciplinary open access archive for the deposit and dissemination of scientific research documents, whether they are published or not. The documents may come from teaching and research institutions in France or abroad, or from public or private research centers.
L'archive ouverte pluridisciplinaire HAL, est destinée au dépôt et à la diffusion de documents scientifiques de niveau recherche, publiés ou non, émanant des établissements d'enseignement et de recherche français ou étrangers, des laboratoires publics ou privés. 


\title{
Journal Name
}

\section{ARTICLE TYPE}

Cite this: DOI: $10.1039 / x x x x x x x x x x$

\section{Large Amplitude Motion within Acetylene-Rare Gas Complexes Hosted in Helium Droplets}

\author{
Marc Briant, ${ }^{*} a^{\dagger}$ Alexandra Viel, ${ }^{* b \ddagger}$ Ephriem Mengesha, ${ }^{\dagger}$ Marc-André Gaveau, ${ }^{\dagger}$ \\ Benoît Soep,$^{\dagger}$ Jean-Michel Mestdagh, ${ }^{\dagger}$ Pierre Jamet, ${ }^{\ddagger}$ Jean-Michel Launay, ${ }^{\ddagger}$ and Li- \\ onel Poisson, ${ }^{\dagger}$
}

Received Date

Accepted Date

DOI: $10.1039 / x x x x x x x x x x$

Near-infrared spectroscopy of the $\mathrm{C}_{2} \mathrm{H}_{2}-\mathrm{Ar}, \mathrm{Kr}$ complexes was performed in the spectral region

www.rsc.org/journalname overlapping the $v_{3} / v_{2}+v_{4}+v_{5}$ Fermi-type resonance of $\mathrm{C}_{2} \mathrm{H}_{2}$. The experiment was conducted along the HElium NanoDroplet Isolation (HENDI) technique in order to study the coupling dynamics between a floppy molecular system $\left(\mathrm{C}_{2} \mathrm{H}_{2}-\mathrm{Ar}\right.$ and $\left.\mathrm{C}_{2} \mathrm{H}_{2}-\mathrm{Kr}\right)$ and a mesoscopic quantum liquid (the droplet). Calculations were performed using a spectral element based close-coupling program and state-of-the-art 2-dimensional potential energy surfaces to determine the bound states of the $\mathrm{C}_{2} \mathrm{H}_{2}-\mathrm{Ar}$ and $\mathrm{C}_{2} \mathrm{H}_{2}-\mathrm{Kr}$ complexes and simulate the observed spectra. This furnished a quantitative basis to unravel how the superfluid and non-superfluid components of the droplet affect the rotation and the deformation dynamics of the hosted complex.

\section{Introduction}

Helium nanodroplet isolation (HENDI) is a well established technique to conduct infrared absorption spectroscopy with rotational resolution on molecular species which are hosted in the droplets. ${ }^{1,2}$ Although helium droplets have a strong superfluid character, molecular rotations within droplets are perturbed. When simulating rovibrational absorption spectra in this context, a simple way is to replace the gas phase rotational and centrifugal correction constants of the embedded molecule by effective constants. ${ }^{3}$ This phenomenon has been rationalized as the development of a non-superfluid component about the molecule under rotation. A series of diffusion and path integral Monte Carlo calculations reviewed by Kwon et al. substantiates this picture. ${ }^{4}$

Close-shell linear molecules are bonded by weak van der Waals forces and form floppy complexes with rare gas atoms. AcetyleneRare Gas $\left(\mathrm{C}_{2} \mathrm{H}_{2}-\mathrm{RG}\right)$ pairs are representative examples. Their floppyness is associated with both the stretching of the $\mathrm{C}_{2} \mathrm{H}_{2}-\mathrm{RG}$ bond and the rotation of $\mathrm{C}_{2} \mathrm{H}_{2}$ moiety with respect to the axis connecting RG to the centre-of-mass (CM) of $\mathrm{C}_{2} \mathrm{H}_{2}$. This leads to a series of bound states, within the well of the $\mathrm{C}_{2} \mathrm{H}_{2}-\mathrm{RG}$ ground state surface. When such a $\mathrm{C}_{2} \mathrm{H}_{2}-\mathrm{RG}$ complex is embedded in

\footnotetext{
† LIDYL, CEA, CNRS, Université Paris-Saclay, CEA Saclay, F-91191 Gif-sur-Yvette, France.

‡Univ Rennes, CNRS, IPR (Institut de Physique de Rennes) - UMR 6251, F-35000 Rennes, France

${ }^{a}$ E-mail: marc.briant@cea.fr

${ }^{b}$ E-mail: alexandra.viel@univ-rennes1.fr
}

a helium droplet, both the rotation and stretching movements responsible for its deformation are perturbed by the helium environment and the associated bound states are shifted when compared to the corresponding states of the free complex. This can be viewed as a benchmark for studying the coupling between a small quantum system (the $\mathrm{C}_{2} \mathrm{H}_{2}-\mathrm{RG}$ complex) with a nanoscale quantum liquid (the droplet). This issue was addressed in the Saclay laboratory for the $\mathrm{C}_{2} \mathrm{H}_{2}-\mathrm{Ne}$ complex in a work that associates HENDI spectroscopy with a theoretical modeling of the $\mathrm{C}_{2} \mathrm{H}_{2}-\mathrm{Ne}$ deformation dynamics. ${ }^{5}$ Hereafter, this work is referred to as $\mathrm{Pa}$ per I. It explored the deformation of the complex when the $\mathrm{C}_{2} \mathrm{H}_{2}$ moiety is vibrationally excited in the vicinity of the $v_{3} / v_{2}+v_{4}+v_{5}$ Fermi dyad. This dyad has been fully analysed by Vanderauwera et al. in free $\mathrm{C}_{2} \mathrm{H}_{2} \cdot{ }^{6}$

The $\mathrm{C}_{2} \mathrm{H}_{2}-\mathrm{Ne}$ complex is characterized by a very weak interaction between $\mathrm{C}_{2} \mathrm{H}_{2}$ and $\mathrm{Ne}$ with little anisotropy. The rotation of $\mathrm{C}_{2} \mathrm{H}_{2}$ within the complex is therefore fairly free. Nevertheless, Paper I showed that the effect of the helium environment on the internal deformation of this complex does not reduce to a perturbation of the sole rotation of $\mathrm{C}_{2} \mathrm{H}_{2}$. The presence of Ne changes in a subtle way the distribution and extension of the non-superfluid component of the helium droplet about $\mathrm{C}_{2} \mathrm{H}_{2}$ as well as the coupling between the $\mathrm{C}_{2} \mathrm{H}_{2}$ rotation and the droplet.

The present work investigates further the dynamical effects of the helium droplet environment on the large amplitude deformation of $\mathrm{C}_{2} \mathrm{H}_{2}-\mathrm{RG}$ complexes. Here, Ne is replaced by Ar and $\mathrm{Kr}$. The $\mathrm{C}_{2} \mathrm{H}_{2}-\mathrm{Ar}$ and $\mathrm{C}_{2} \mathrm{H}_{2}-\mathrm{Kr}$ complexes are considered, again in a 
joint experimental and theoretical approach. As we shall see, the $v_{3} / v_{2}+v_{4}+v_{5}$ Fermi resonance is still at play in these complexes, which are therefore explored in the vicinity of the corresponding Fermi dyad. Two kinds of effects are expected when neon is replaced by the heavier and more polarizable argon and krypton atoms. First, the $\mathrm{C}_{2} \mathrm{H}_{2}-\mathrm{Ar}$, $\mathrm{Kr}$ binding energies and anisotropies are larger. ${ }^{7-10}$ Accordingly, the $\mathrm{C}_{2} \mathrm{H}_{2}-\mathrm{Ar}$, $\mathrm{Kr}$ complexes are likely more rigid than $\mathrm{C}_{2} \mathrm{H}_{2}-\mathrm{Ne}$ and their bound states are expected to deviate from pure rotational states of $\mathrm{C}_{2} \mathrm{H}_{2}$. Hence, assumptions of the theoretical treatment in Paper I do not apply here. This concerns the oscillator strength of the ro-vibrational transitions. They are deduced from Hönl-London factors in Paper I whereas here, they are calculated explicitly. Second, the larger polarizability of the heavy rare gases is expected to increase substantially the extension of the non-superfluid component about the hosted complex. This may affect dramatically the energy of the excited bound states of the complex. Exploring these two effects make the centre of the present work.

\section{Experimental setup}

The experimental setup has been described extensively in Paper I. It associates a molecular beam carrying helium droplets (average size of a few thousands atoms), two pickup regions where the desired species are deposited on the droplets $\left(\mathrm{C}_{2} \mathrm{H}_{2}\right.$ first, then $\mathrm{Ar}$ or $\mathrm{Kr}$ ). A tunable infrared optical parametric oscillator laser is passing through a multi-pass mirror assembly and illuminates the droplet beam.

The binding energy of the $\mathrm{C}_{2} \mathrm{H}_{2}-\mathrm{Ar}, \mathrm{Kr}$ complexes $\left(122.93 \mathrm{~cm}^{-1}\right.$ and $150.46 \mathrm{~cm}^{-1}$, respectively 9,10$)$ is much larger than the droplet temperature $\left(0.37 \mathrm{~K} \text {, i.e. } 0.26 \mathrm{~cm}^{-1}\right)^{11}$ and the He-He and $\mathrm{C}_{2} \mathrm{H}_{2}-\mathrm{He}$ well depths $\left(\mathrm{D}_{e}=7.4 \mathrm{~cm}^{-1}\right.$ and $24.22 \mathrm{~cm}^{-1}$, respectively ${ }^{7,12}$ ). Hence, when both a single $\mathrm{C}_{2} \mathrm{H}_{2}$ molecule and a single rare gas atom are present in the same droplet, they associate together and form a 1:1 complex $\left(\mathrm{C}_{2} \mathrm{H}_{2}-\mathrm{Ar}\right.$ or $\mathrm{C}_{2} \mathrm{H}_{2}-\mathrm{Kr}$ whether Ar or $\mathrm{Kr}$ is picked-up).

The experiment consists in monitoring the flux of helium droplets while scanning the laser frequency. Each time the laser frequency hits a ro-vibrational transition of the hosted species, a ro-vibrational excitation occurs. Subsequently, the excess energy relaxes towards the droplet, a partial (or total) droplet evaporation occurs and the flux of helium droplets decreases. The latter is monitored using a mass spectrometer tuned to the $\mathrm{He}_{2}^{+}$mass. Accordingly, the decrease of the $\mathrm{He}_{2}^{+}$signal reflects directly the absorption spectrum of the hosted species. Here, the laser frequency is scanned between 3279 and $3310 \mathrm{~cm}^{-1}$, a range which overlaps the two dyads due to the $v_{3}, v_{2}+v_{4}+v_{5}$ Fermi resonance of the $\mathrm{C}_{2} \mathrm{H}_{2}$ molecule.

The pick-up process used to deposit $\mathrm{C}_{2} \mathrm{H}_{2}$ and the rare gas partner on the droplet is stochastic. Only the average number of each species per droplet is determined by the gas pressure in the pickup region. When adjusting this pressure low enough, the number of droplet carrying more than one $\mathrm{C}_{2} \mathrm{H}_{2}$ molecule and more than one rare gas atom is made very small. This number is given by the Poisson statistics which is followed approximately by the pickup process ${ }^{13}$. Under the present experimental condition, droplets which can absorb the IR laser light are believed to carry a single
$\mathrm{C}_{2} \mathrm{H}_{2}$ molecule, a 1:1 $\mathrm{C}_{2} \mathrm{H}_{2}-\mathrm{RG}$ complex or the $\left(\mathrm{C}_{2} \mathrm{H}_{2}\right)_{2}$ dimer. The number of those carrying larger complexes is considered as negligible.

\section{Simulation}

The simulation is split in 3 steps. Step (i) determines the energy and wavefunctions of the bound states of the rotating $\mathrm{C}_{2} \mathrm{H}_{2}-\mathrm{RG}$ complex in the ground state. The coupling between the complex and the helium droplet is treated phenomelogically by giving effective values to several dynamical input parameters of the bound state calculation (see below). The latter describe both the overall rotation of the complex and its deformations along two coordinates: the $\mathrm{C}_{2} \mathrm{H}_{2}-\mathrm{RG}$ radius and the frustrated rotation of $\mathrm{C}_{2} \mathrm{H}_{2}$ within the complex. Step (ii) calculates the oscillator strength of the transitions corresponding to the excitation of the $\mathrm{C}_{2} \mathrm{H}_{2}$ moiety of the complex within the $v_{3} / v_{2}+v_{4}+v_{5}$ Fermi dyad. As in Paper I, the bound state structure is assumed to be the same whether the dimer is in the ground state or vibrationally excited within the $v_{3} / v_{2}+v_{4}+v_{5}$ Fermi dyad of the $\mathrm{C}_{2} \mathrm{H}_{2}$ moiety. Accordingly, the energy levels and wavefunctions found at step $i$ ) can be used directly to calculate the desired oscillator strengths. Step (iii) simulates the absorption spectrum of the complex as a sum over the transitions obtained at step (ii).

Step (i) - The bound states of the $\mathrm{C}_{2} \mathrm{H}_{2}-\mathrm{RG}$ complex are derived by solving the Schrödinger equation which describes the movement of $\mathrm{C}_{2} \mathrm{H}_{2}$ with respect to the rare gas. $\mathrm{C}_{2} \mathrm{H}_{2}$ is described as a pseudo rigid linear rotor with rotational and centrifugal correction constants $B$ and $D$. This reduces the dimensionality of the problem to two internal coordinates $[\mathrm{R}, \theta]$ : $\mathrm{R}$ is the distance between the rare gas and the centre-of-mass (CM) of $\mathrm{C}_{2} \mathrm{H}_{2} ; \theta$ is the alignment angle between the symmetry axis of $\mathrm{C}_{2} \mathrm{H}_{2}$ and the axis which links the rare gas to the $\mathrm{CM}$ of $\mathrm{C}_{2} \mathrm{H}_{2}$. The calculations are performed using the spectral element based close-coupling program VRBoundScat developed in the group of Jean-Michel Launay ${ }^{14-16}$ and the $\mathrm{C}_{2} \mathrm{H}_{2}-\mathrm{Ar}$, Kr potential energy surfaces $\mathrm{V}(\mathrm{R}, \theta)$ derived in the group of Jacky Liévin. ${ }^{9,10}$ This provides us with bound states and wave functions. The latter are labeled $|\alpha, \mathrm{J}, \mathrm{M}\rangle$ where $\alpha$ is the state number in the $\{\mathrm{J}, \mathrm{M}\}$ multiplicity, $\mathrm{J}$ being the total orbital angular momentum of the complex and $\mathrm{M}$ its projection in the laboratory. The location of these levels within the well of the potential energy surface is controlled by three dynamical parameters: the reduced mass $\mu$ of the $\mathrm{C}_{2} \mathrm{H}_{2}-\mathrm{RG}$ system and the $\mathrm{B}$ and $\mathrm{D}$ constants mentioned above. The difference with the equivalent calculations in Paper I is that VRBoundScat provides us very simply with energy levels and wavefunctions. Transition strengths are then easy to calculate explicitly.

Step (ii) - Given the assumption mentioned above, $|\alpha, \mathrm{J}, \mathrm{M}\rangle$ describes either a bound level of the vibrational ground state $(\mid \alpha$ ",J",M" $\rangle)$ or a bound level within one component of the $v_{3} / v_{2}+v_{4}+v_{5}$ Fermi dyad $\left(\left|\alpha^{\prime}, \mathrm{J}^{\prime}, \mathrm{M}^{\prime}\right\rangle\right)$. Accordingly, the force of the transitions which are at play in the present experiment are proportional to:

$$
S_{J^{\prime \prime} J^{\prime}}=\sum_{M^{\prime \prime}} \sum_{M^{\prime}}\left|\left\langle\alpha^{\prime \prime}, J^{\prime \prime}, M^{\prime \prime}|\vec{\mu}| \alpha^{\prime}, J^{\prime}, M^{\prime}\right\rangle\right|^{2}
$$

Step (iii) - Standard techniques (e.g. Chapter 5 in the Bernath 
textbook $\left.{ }^{17}\right)$ allow us to derive the absorption profile associated with each $\left\langle\alpha^{\prime}, J^{\prime}, M^{\prime}|\leftarrow| \alpha^{\prime \prime}, J^{\prime \prime}, M^{\prime \prime}\right\rangle$ transition. The full absorption spectrum is given by the sum over $\alpha$ ", J" and M", taking into account the proper weighting of the ground energy levels at the droplet temperature $(0.37 \mathrm{~K})$.

The same strategy as in Paper I is followed here to quantify the effect of the helium environment on the deformation dynamics of the complex. A best fit of the experimental spectrum is performed by adjusting the three dynamical parameters $\mu, \mathrm{B}$ and $\mathrm{D}$ and the two excitation frequencies $v_{U D}$ and $v_{L D}$ towards the upper and lower components of the $v_{3} / v_{2}+v_{4}+v_{5}$ Fermi dyad. The three former appear in the close-coupling calculation and the two latter in the spectrum simulation.

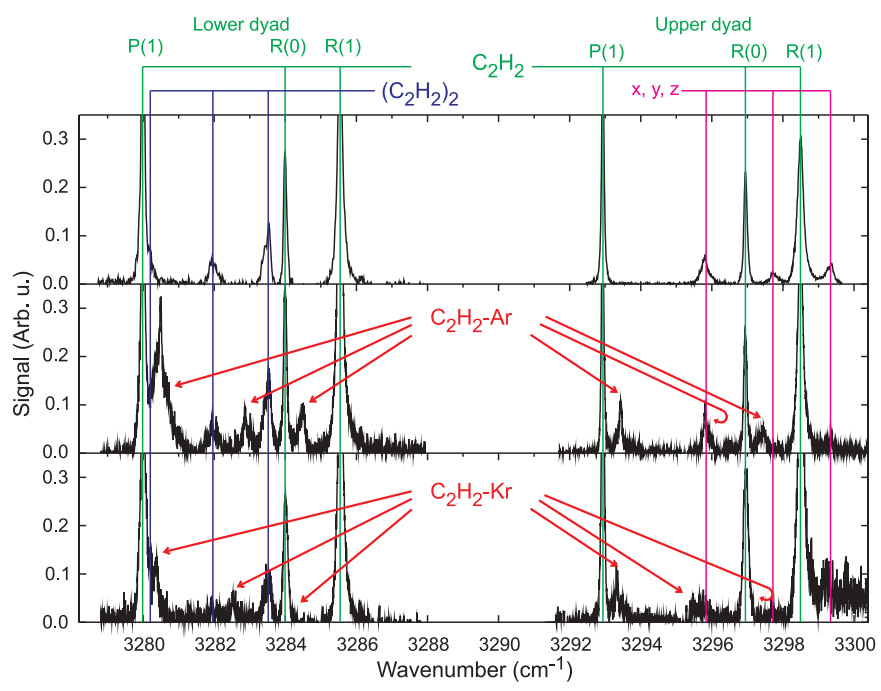

Fig. 1 Spectrum recorded in the present $\mathrm{C}_{2} \mathrm{H}_{2}-\mathrm{Ar}$ (middle panel) and $\mathrm{C}_{2} \mathrm{H}_{2}-\mathrm{Kr}$ (bottom panel) experiments compared to that reported in Paper I (top panel) when no rare gas is present in the pickup region.

\section{Experimental Results}

The spectra recorded in the present experiment are shown in Fig. 1 (middle and bottom panels). They are compared with a spectrum taken from paper I (top panel) where no rare gas was present in the pick-up region.

The spectrum shown in the upper panel is due specifically to the absorption by droplets carrying no $\mathrm{C}_{2} \mathrm{H}_{2}-\mathrm{RG}$ gas complex. In this spectrum, the two series of intense narrow peaks labeled $\mathrm{P}(1), \mathrm{R}(0)$ and $\mathrm{R}(1)$ in green are associated with droplets carrying a single $\mathrm{C}_{2} \mathrm{H}_{2}$ molecule. They reflect the absorption of acetylene within the two dyads created by the $v_{3} / v_{2}+v_{4}+v_{5}$ Fermi resonance. This assignment follows that provided by Nauta and Miller who report the first HENDI experiment on acetylene. ${ }^{18}$ The three bands marked in blue in the lower dyad region are associated with droplets carrying a $\left(\mathrm{C}_{2} \mathrm{H}_{2}\right)_{2}$ dimer. They were first observed by Nauta and Miller and assigned to $K_{a}(2 \leftarrow 1), K_{a}(1 \leftarrow 0)$ and $K_{a}(0 \leftarrow 1)$ transitions in an asymmetric top representation of the dimer. ${ }^{18}$ They were fully analysed more recently by Briant $e t a l$. in terms of a tunneling isomerization process which exchanges the two $\mathrm{C}_{2} \mathrm{H}_{2}$ molecules. Finally, three features labeled $\mathrm{x}, \mathrm{y}$ and $\mathrm{z}$ in the figure (pink labeling in the upper dyad region) were ob- served also in paper I. Their origin is not clear and could be either combination bands in $\left(\mathrm{C}_{2} \mathrm{H}_{2}\right)_{>2}$ multimers or due to impurities.

The other bands which appear in the middle and bottom panels of the figure correspond to transitions of the $1: 1 \mathrm{C}_{2} \mathrm{H}_{2}-\mathrm{Ar}$ (middle panel) or $\mathrm{C}_{2} \mathrm{H}_{2}-\mathrm{Kr}$ (bottom panel) complexes carried by a helium droplet. They are indicated by red arrows. Several of them have an accidental coincidence with lines observed in the top spectrum where no rare gas atom is present in the pick-up. Only a careful comparison with simulated spectra (done below) can confirm the assignment.

\section{Simulation results}
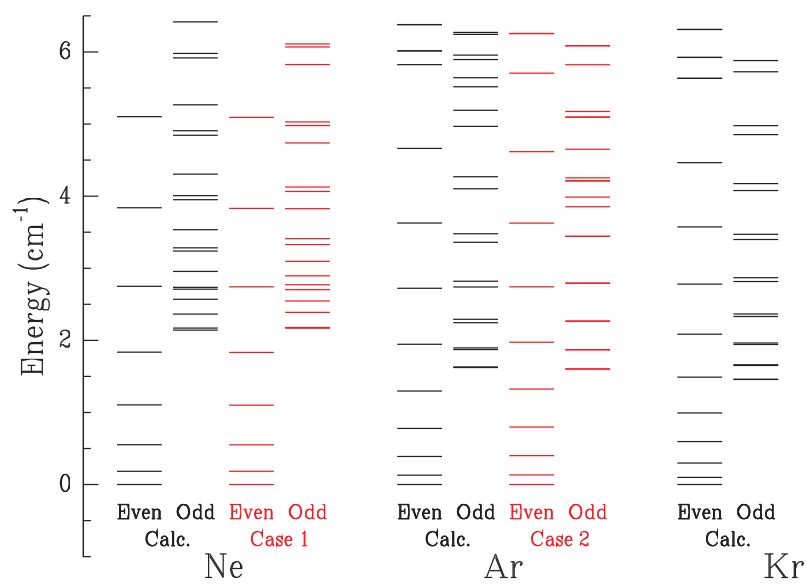

Fig. 2 Bound energy levels of the free $\mathrm{C}_{2} \mathrm{H}_{2}-\mathrm{Ar}, \mathrm{Kr}$ complexes compared to those of $\mathrm{C}_{2} \mathrm{H}_{2}-\mathrm{Ne}$. The black columns report the present calculations. The red columns correspond to the cases 1 and 2 of the Hutson model recalled in the text. Labels odd and even refer to the nuclear spin parity when the $\mathrm{H}$-atom of $\mathrm{C}_{2} \mathrm{H}_{2}$ are exchanged (ortho/para symmetry of $\mathrm{C}_{2} \mathrm{H}_{2}$ ). The even levels of lowest energy are taken as energy origin for each rare gas.

\subsection{Spectra of free 1:1 complexes.}

Before simulating the spectra of the $1: 1 \mathrm{C}_{2} \mathrm{H}_{2}-\mathrm{RG}$ complexes in the helium droplet environnement, it is interesting to examine the predictions of our model when the $\mathrm{C}_{2} \mathrm{H}_{2}-\mathrm{Ne}$, $\mathrm{Ar}$ and $\mathrm{Kr}$ complexes are free of any environment. Interesting also will be to compare these predictions with the available litterature when available as it is the case with the $\mathrm{C}_{2} \mathrm{H}_{2}$-Ar complex.

The predicted bound states of the free $1: 1 \mathrm{C}_{2} \mathrm{H}_{2}-\mathrm{Ne}, \mathrm{Ar}$ and $\mathrm{Kr}$ complexes are shown in Fig. 2 using the dynamical parameters $\mu, \mathrm{B}$ and D listed in Tab. 1. Those calculated for Ne and Ar are compared in the same figure to the predictions of simple models presented in the discussion below.

The comparison with the available litterature on the $\mathrm{C}_{2} \mathrm{H}_{2}-\mathrm{Ar}$ complex is achieved in Fig. 3 which shows the present simulation (black curve) and that of Bemish et al. in the spectral region corresponding to the upper dyad of acetylene. ${ }^{22}$ Note that in their simulation, Bemish et al. have adjusted the shape of the $\mathrm{C}_{2} \mathrm{H}_{2}-\mathrm{Ar}$ potential energy surface to ensure that their simulated spectrum nicely reproduces the experimental one recorded in the same work. Here, no such adjustment is done and the $\mathrm{V}(\mathrm{R}, \theta)$ potential energy surface of Lauzin et al. is used directly. ${ }^{10}$ 


\begin{tabular}{|c|c|c|c|c|c|c|c|c|}
\hline & \multicolumn{4}{|c|}{ Isolated } & \multicolumn{4}{|c|}{ He-droplet } \\
\hline & $\mathrm{C}_{2} \mathrm{H}_{2}{ }^{(a)}$ & $\mathrm{C}_{2} \mathrm{H}_{2}-\mathrm{Ne}^{(b)}$ & $\mathrm{C}_{2} \mathrm{H}_{2}-\mathrm{Ar}^{(c)}$ & $\mathrm{C}_{2} \mathrm{H}_{2}-\mathrm{Kr}^{(c)}$ & $\mathrm{C}_{2} \mathrm{H}_{2}{ }^{(d)}$ & $\mathrm{C}_{2} \mathrm{H}_{2}-\mathrm{Ne}^{(d)}$ & $\mathrm{C}_{2} \mathrm{H}_{2}-\mathrm{Ar}^{(c)}$ & $\mathrm{C}_{2} \mathrm{H}_{2}-\mathrm{Kr}^{(c)}$ \\
\hline$\mu(\mathrm{u})$ & & 11.3049 & 15.7552 & 19.8524 & & $15.5 \pm 1$ & $33 \pm 5$ & $40 \pm 10$ \\
\hline $\mathrm{B}\left(\mathrm{cm}^{-1}\right)$ & 1.1766455 & $1.176 / 1.172$ & 1.1766455 & 1.1766455 & 1.04 & $1.140 \pm 0.005$ & $1.09 \pm 0.02$ & $1.09 \pm 0.02$ \\
\hline $\mathrm{D}\left(\mathrm{cm}^{-1}\right)$ & $1.62 \times 10^{-6}$ & & $1.62 \times 10^{-6}$ & $1.62 \times 10^{-6}$ & 0.019 & $0.0194 \pm 0.005$ & $0.042 \pm 0.005$ & $0.042 \pm 0.005$ \\
\hline $\mathrm{H}\left(\mathrm{cm}^{-1}\right)$ & & & & & & & 0.00055 & 0.00055 \\
\hline $\mathrm{G}_{L D}\left(\mathrm{~cm}^{-1}\right)$ & 3281.90062 & 3281.8991 & 3281.52 & & 3281.99 & $3282.07 \pm 0.02$ & $3281.66 \pm 0.02$ & $3281.44 \pm 0.02$ \\
\hline $\mathrm{G}_{U D}\left(\mathrm{~cm}^{-1}\right)$ & 3294.841 & 3294.8395 & 3294.46 & & 3294.94 & $3295.01 \pm 0.02$ & $3294.61 \pm 0.02$ & $3294.39 \pm 0.02$ \\
\hline
\end{tabular}

Table 1 Reduced mass $\mu$ and spectroscopic constants B, D, $\mathrm{G}_{L D}$ and $\mathrm{G}_{U D}$ used to simulate the spectra. ${ }^{(a)}$ Taken from Ref. ${ }^{18}$. ${ }^{(b)}$ Taken from Ref. ${ }^{20}$. ${ }^{(c)}$ present work. ${ }^{(d)}$ Taken from Ref. ${ }^{5}$.

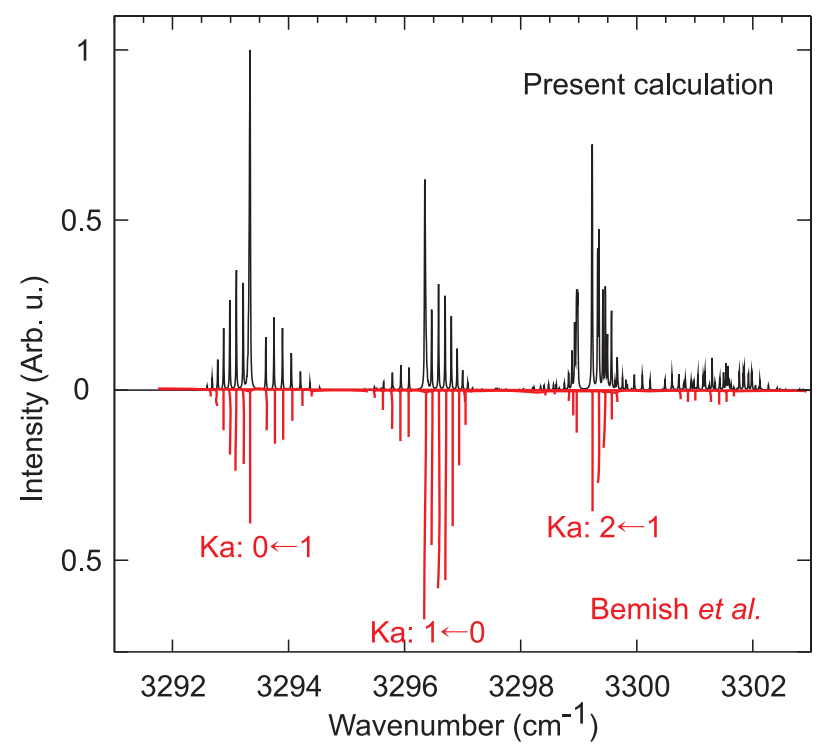

Fig. 3 Spectrum of the free $1: 1 \mathrm{C}_{2} \mathrm{H}_{2}-$ Ar complex simulated in the present work (in black) compared with the simulated one reported by Bemish et al.. ${ }^{22}$ Only the upper dyad region is shown. The band assignments are those of Bemish et al., following an asymmetric top $\left|J K_{a} K_{c}\right\rangle$ description of the complex.

\subsection{Simulated spectrum of the $\mathrm{C}_{2} \mathrm{H}_{2}-\mathrm{Ar}$, $\mathrm{Kr}$ complexes}

Because of the Poisson statistics of the pickup, a fraction of the droplet present in the $\mathrm{C}_{2} \mathrm{H}_{2}-\mathrm{Ar}, \mathrm{Kr}$ experiments carry no rare gas atom. The corresponding absorption spectrum is thus due to the acetylene monomer, dimer and, as discussed in paper I, higher multimers or impurities for the $\mathrm{x}, \mathrm{y}$ and $\mathrm{z}$ bands. The data reported in Fig. 1 when acetylene only is present in the pickup (top panel) can be used to extract the $\mathrm{C}_{2} \mathrm{H}_{2}$-only contribution from the spectrum shown in the lower panels. This cannot be performed directly since the experiment were not run under the same experimental condition. The relative contribution of the monomer, dimer and larger multimers of $\mathrm{C}_{2} \mathrm{H}_{2}$ need to be adjusted in each experiment. The result is shown in Figs. 4 and 5 for the Ar and $\mathrm{Kr}$ experiments, respectively. Of course, the subtraction is not very accurate underneath the intense $\mathrm{C}_{2} \mathrm{H}_{2}$ bands that show up in the dot green curve.

The red curve in the same figure shows the simulated spectrum which best fits the experiment, using the parameters shown in the last columns of Tab. 1 .

The large value of $\mathrm{D}$ which appears in Tab. 1 causes an arte-

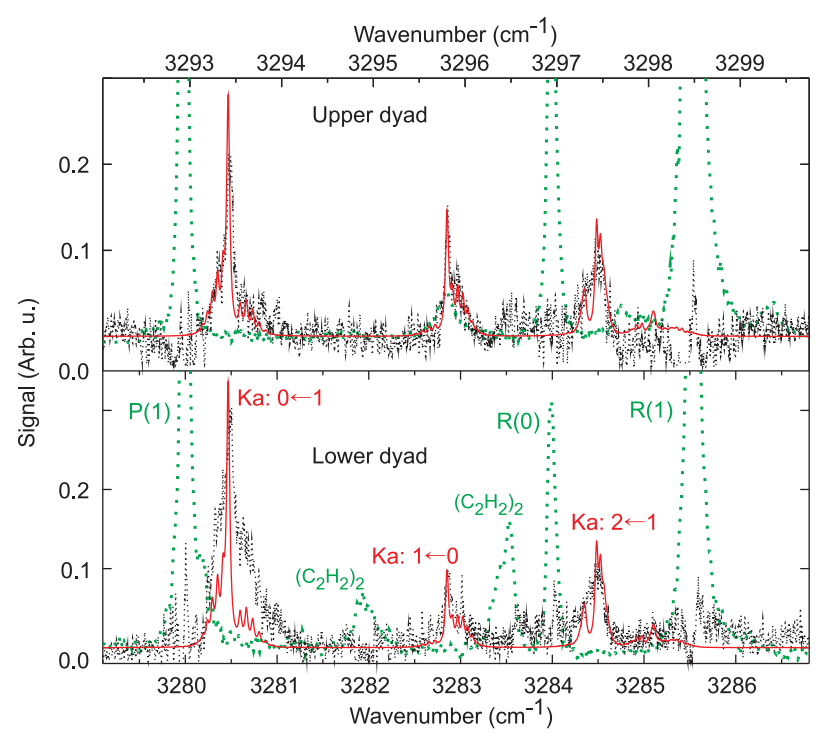

Fig. 4 Comparison between the experimental (black dots) and simulated spectra (red curve) for the $\mathrm{C}_{2} \mathrm{H}_{2}-\mathrm{Ar}$ complex. The green dotted curve shows the acetylene-only signal which was subtracted to get the black dotted curve. The green labels are taken from Fig. 1. The red one are discussed in the text.

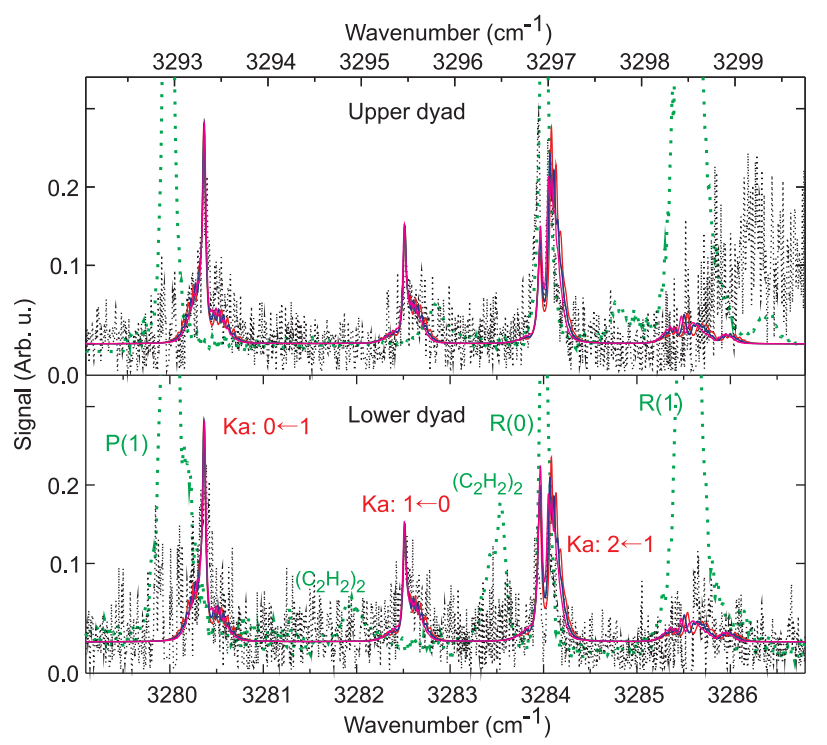

Fig. 5 Same caption as Fig. 4 for the $\mathrm{C}_{2} \mathrm{H}_{2}-\mathrm{Kr}$ complex.

fact with the angular basis vectors built from $\mathrm{C}_{2} \mathrm{H}_{2}$ rotation lev- 
els of high j. For a reliable calculation of the bound state of the complex, this basis must include vectors of large $j$ values. Unfortunately when $\mathrm{j}>8$, the $-\mathrm{Dj}^{2}(\mathrm{j}+1)^{2}$ term leads to decreasing energies of the rotational levels as $\mathrm{j}$ is increased. To bypass this trouble, a sextic centrifugal distortion constant $\mathrm{H}=5.510^{-4} \mathrm{~cm}^{-1}$ is added to built the basis vectors. As a side effect, $\mathrm{H}$ appears in the calculation as a further dynamical parameter which acts in a similar way as B and D. In the asymmetric top representation of the complex, the value chosen for $\mathrm{H}$ makes that only the energy of $\mathrm{K}_{a}>2$ states is affected. Given the droplet temperature $(0.37 \mathrm{~K})$ these levels do not participate strongly to the observed spectrum. The use of $\mathrm{H}$ thus appears as technical only and its value does not need to be discussed.

\section{Discussion}

Two effects are pointed out in the introduction regarding the replacement of $\mathrm{Ne}$ by a heavier atom in $\mathrm{C}_{2} \mathrm{H}_{2}-\mathrm{RG}$ complexes. The first one is related to the enhanced rigidity of the complex. It is examined in the light of our calculations on free complexes. The second effect is associated with the coupling dynamics between the complex, the non-superfluid and the superfluid components of the droplet. It is examined below by discussing the effective constants B and D derived in the previous section when fitting the observed spectra.

\subsection{Bound energy levels of the free complexes}

We anticipated in the introduction that the larger polarizability of $\mathrm{Ar}$ and $\mathrm{Kr}$ makes the $\mathrm{C}_{2} \mathrm{H}_{2}-\mathrm{Ar}$, $\mathrm{Kr}$ complexes more rigid than $\mathrm{C}_{2} \mathrm{H}_{2}-\mathrm{Ne}$. A quick look to Fig. 2 shows that the overall distributions of the $\mathrm{C}_{2} \mathrm{H}_{2}-\mathrm{Ar}$ and $\mathrm{C}_{2} \mathrm{H}_{2}-\mathrm{Kr}$ levels are quite similar whereas that of $\mathrm{C}_{2} \mathrm{H}_{2}-\mathrm{Ne}$ is a little different. This point is examined by comparing the bound state energies derived from the full calculation on the free $\mathrm{C}_{2} \mathrm{H}_{2}-\mathrm{Ne}$ and $\mathrm{C}_{2} \mathrm{H}_{2}-$ Ar complexes (in black in Fig. 2) to those derived from two simple models (in red in the same figure) built on different assumptions regarding the rigidity of the complexes.

Hutson examined exhaustively how the rotational states of a linear molecule like $\mathrm{C}_{2} \mathrm{H}_{2}$ are perturbed and split when the molecule interacts with a rare gas atom within a van der Waals complex. ${ }^{21}$ Hutson identified several situations, named cases 1 , 2 and 3 when comparing the rotational constant of the linear molecule, that describing the overall rotation of the complex and an anisotropy term $V_{2}$, where $V_{2}$ is the second order term in the Legendre expansion of the interaction potential as a function of the bending angle of the complex. This classification reproduces that pointed out earlier by Bratož and Martin. ${ }^{23}$

The relevant case for the $\mathrm{C}_{2} \mathrm{H}_{2}-\mathrm{Ne}$ complex is "Case 1 ". It is adapted to a small anisotropy of the interaction potential and therefore describes non-rigid complexes. The corresponding bound state energies are given by Eqs. (26) and (52) in Hutson's work. ${ }^{21}$ Five parameters appear in the model: the rotation and centrifugal correction constants of free $\mathrm{C}_{2} \mathrm{H}_{2}$ (given in Tab. 1), the corresponding constants for the overall rotation of the complex (taken to be 0.092 and $1.910^{-5} \mathrm{~cm}^{-1}$, respectively) and the anisotropy term $\mathrm{V}_{2}$ (taken to be $0.87 \mathrm{~cm}^{-1}$ ). The corresponding results are shown in red in the "case 1" column of Fig. 2. A quantitative agreement is observed, especially for the even states. The fact that the model assumes an exactly $\mathrm{T}$ shape complex and describes the interaction potential by the sole second order Legendre expansion term is certainly the origin of the deviations observed for the odd states at energies larger than $3.5 \mathrm{~cm}^{-1}$. Nevertheless, the observed agreement confirms quantitatively the non rigid character of the $\mathrm{C}_{2} \mathrm{H}_{2}-\mathrm{Ne}$ complex since the anisotropy term $\mathrm{V}_{2}$ is smaller than the rotation constant of $\mathrm{C}_{2} \mathrm{H}_{2}\left(0.87 \mathrm{~cm}^{-1}\right.$ against $\left.1.176 \mathrm{~cm}^{-1}\right)$ and therefore does not hinder completely the rotation of $\mathrm{C}_{2} \mathrm{H}_{2}$ within the complex. Bemish et al. came to the same conclusion when relating their calculation on the $\mathrm{C}_{2} \mathrm{H}_{2}-\mathrm{Ne}$ complex to the coupling schemes pointed out by Bratož and Martin. ${ }^{20,23}$

Cases 2 and 3 in Hutson's work describe situations where the anisotropy term $\mathrm{V}_{2}$ severely (case 2) or totally (case 3) hinders the $\mathrm{C}_{2} \mathrm{H}_{2}$ rotation. The case 2 column of Fig. 2 is calculated using Eqs. (34) and (55) of Hutson's work. ${ }^{21}$ The same five parameters as above are involved, of course with different values. With the $\mathrm{C}_{2} \mathrm{H}_{2}-\mathrm{Ar}$ complex, the overall rotation and centrifugal correction constants become 0.067 and $4.010^{-5} \mathrm{~cm}^{-1}$, respectively and $\mathrm{V}_{2}$ is taken to be $3.75 \mathrm{~cm}^{-1}$. Given the limitations of the model that have been mentioned already, a satisfactory agreement is observed Fig. 2 with the full calculation (black column). This confirms quantitatively the anticipation that the $\mathrm{C}_{2} \mathrm{H}_{2}-\mathrm{Ar}$ complex is much more rigid than $\mathrm{C}_{2} \mathrm{H}_{2}-\mathrm{Ne}$. The value of $\mathrm{V}_{2}=3.75 \mathrm{~cm}^{-1}$ is indeed substantially larger than the rotational constant of $\mathrm{C}_{2} \mathrm{H}_{2}$ $\left(1.176 \mathrm{~cm}^{-1}\right)$ and the deformation of the $\mathrm{C}_{2} \mathrm{H}_{2}-\mathrm{Ar}$ complex, although not blocked, is severely limited. Given the ressemblance between their level distribution, the $\mathrm{C}_{2} \mathrm{H}_{2}-\mathrm{Ar}$ and $\mathrm{C}_{2} \mathrm{H}_{2}-\mathrm{Kr}$ complexes fall likely in the same category regarding their rigidity, i.e. case 2 of Hutson.

This was actually the conclusion drawn by Bemish et al. for the $\mathrm{C}_{2} \mathrm{H}_{2}-$ Ar complex, in a joint experimental and theoretical work that has already been quoted. ${ }^{22}$ These authors have explored the same $v_{3} / v_{2}+v_{4}+v_{5}$ Fermi dyad as here, derived a $\mathrm{C}_{2} \mathrm{H}_{2}-\mathrm{Ar}$ potential energy surface "upon which the complex executes wide amplitude bending motion" and optimized this surface to best reproduce the experimental spectrum that they measured. It is very comforting to observe that the absorption spectrum, which these authors simulated (red spectrum in Fig. 3), is in quantitative agreement with that derived in the present work (black spectrum in the same figure) from the potential energy surface of Lauzin et al. ${ }^{10}$

\subsection{Hindered dynamics of the $\mathrm{C}_{2} \mathrm{H}_{2}-\mathrm{Ar}$, $\mathrm{Kr}$ complexes within a helium droplet}

The black curves in Figs. 4 and 5 report specifically the absorption spectrum of the $\mathrm{C}_{2} \mathrm{H}_{2}-\mathrm{Ar}, \mathrm{Kr}$ complexes in the helium environment when excited in the vicinity of the $v_{3} / v_{2}+v_{4}+v_{5}$ Fermi dyad of acetylene. Three fairly intense bands are observed in each dyad of these complexes. They are assigned and discussed below. We focus here on a fourth band of low intensity, which appears both in the calculation (red curve) and the experiment (black curve, within noise for $\mathrm{C}_{2} \mathrm{H}_{2}-\mathrm{Ar}$ ) in the blue side of each dyad 
of the complexes. It is predicted and observed near 3285.5 and $3298.5 \mathrm{~cm}^{-1}$ for $\mathrm{C}_{2} \mathrm{H}_{2}-\mathrm{Kr}$. It is located accidentally underneath the very intense $\mathrm{R}(1)$ band of $\mathrm{C}_{2} \mathrm{H}_{2}$ and therefore, it is poorly documented experimentally. From the calculation we know that it corresponds to the excitation of the first excited stretch level along the $\mathrm{C}_{2} \mathrm{H}_{2}-\mathrm{RG}$ coordinate.

The three other bands mimic the three bands simulated in Fig. 3 for the free $\mathrm{C}_{2} \mathrm{H}_{2}-$ Ar complex, although their central frequency is different. Bemish et al. assigned these bands as corresponding to the $\mathrm{K}_{a}: 0 \leftarrow 1,1 \leftarrow 0$ and $2 \leftarrow 1$ transitions in an asymmetric top representation $\left|\mathrm{J}, \mathrm{K}_{a}, \mathrm{~K}_{c}\right\rangle$ of the $\mathrm{C}_{2} \mathrm{H}_{2}-\mathrm{Ar}$ complex. ${ }^{22}$ We keep the same assignment and the same notation here for the $\mathrm{C}_{2} \mathrm{H}_{2}-\mathrm{Ar}$, $\mathrm{Kr}$ complexes. If these complexes were perfectly T-shaped, the $\mathrm{K}_{a}$ quantum number would describe an helicopter motion where the "hat" of the T would be the rotor $\left(\mathrm{C}_{2} \mathrm{H}_{2}\right)$ and the "body" the rotation axis $\left(\mathrm{C}_{2} \mathrm{H}_{2}-\mathrm{RG}\right)$. The fact that the odd state $\mathrm{K}_{a}=1$ is populated at the droplet temperature $(0.37 \mathrm{~K})$ reflects that the ortho/para symmetry of the $\mathrm{C}_{2} \mathrm{H}_{2}$ molecule (the hat of the $\mathrm{T}$ ) is preserved in the helium environment. This was already observed in Paper I with the $\mathrm{C}_{2} \mathrm{H}_{2}-\mathrm{Ne}$ complex.

Importantly also, for both the $\mathrm{C}_{2} \mathrm{H}_{2}-\mathrm{Ar}$ and $\mathrm{C}_{2} \mathrm{H}_{2}-\mathrm{Kr}$ complexes, the three bands assigned to $\mathrm{K}_{a}: 0 \leftarrow 1,1 \leftarrow 0$ and $2 \leftarrow 1$ transitions are in exact correspondance between the upper and lower components of the Fermi dyad (the horizontal scale is the same in both panels of Figs. 4 and 5, only the absolute frequency changes).

Whatever the rare gas, the 0-0 transitions $G_{L D}$ and $G_{U D}$ are very close to the corresponding values in the gas phase. This points out that all the energy levels are shifted by the same quantity for a given rare gas. This confirms also what was anticipated in the introduction: the $v_{3} / v_{2}+v_{4}+v_{5}$ Fermi resonance is still at play in the $\mathrm{C}_{2} \mathrm{H}_{2}-\mathrm{Ar}$, $\mathrm{Kr}$ complexes in the helium environment. Its coupling constant is essentially the same as that in $\mathrm{C}_{2} \mathrm{H}_{2}$. This was observed already by Bemish et al. for the free $\mathrm{C}_{2} \mathrm{H}_{2}-\mathrm{Ar}$ complex. ${ }^{22}$

The central issue at this point of the discussion is the comparison between the experimental spectra in Figs. 4 and 5 (black curves) and those simulated (red curve) using the effective parameters $\mu, \mathrm{B}, \mathrm{D}_{L D}, \mathrm{G}_{U D}$ listed in Tab. 1. A satisfactory agreement is observed between the experimental spectra and the simulations.

For $\mathrm{C}_{2} \mathrm{H}_{2}-\mathrm{Ar}$, this agreement concerns all the bands, except that between 3280 and $3281.2 \mathrm{~cm}^{-1}$ in the lower dyad, which was especially difficult to extract. We observe indeed in Fig. 1 that absorptions of three different origins participate to this spectral region. The desired one due to $\mathrm{C}_{2} \mathrm{H}_{2}-\mathrm{Ar}$ is superimposed to that of $\left(\mathrm{C}_{2} \mathrm{H}_{2}\right)_{2}$ in the blue wing of the $\mathrm{C}_{2} \mathrm{H}_{2}$ absorption. Subtraction of the $\left(\mathrm{C}_{2} \mathrm{H}_{2}\right)_{2}$ contribution is made difficult because the $\mathrm{C}_{2} \mathrm{H}_{2}$ only and the $\mathrm{C}_{2} \mathrm{H}_{2} / \mathrm{Ar}$ experiments are not performed under the same pick-up conditions. Since no band assigned to the dimer are observed in the upper dyad, the shape of the corresponding $\mathrm{C}_{2} \mathrm{H}_{2}-\mathrm{Ar}$ band is more reliable when observed in the upper dyad.

With $\mathrm{C}_{2} \mathrm{H}_{2}-\mathrm{Kr}$, the agreement between simulation and experiment is observed for the three $\mathrm{K}_{a}: 0 \leftarrow 1,1 \leftarrow 0$ and $2 \leftarrow 1$ transitions. The $\mathrm{K}_{a}: 2 \leftarrow 1$ band of $\mathrm{C}_{2} \mathrm{H}_{2}-\mathrm{Kr}$ is accidentally underneath the $\mathrm{R}(1)$ transition of $\mathrm{C}_{2} \mathrm{H}_{2}$. Fortunately, the latter is not ex- tremely intense and the subtraction is accurate enough to observe that the shape of the experimental $\mathrm{K}_{a}: 2 \leftarrow 1$ band reproduces that predicted by the calculation in both dyads.

With the restrictions above, the agreement between experiment and simulation in Figs. 4 and 5 can be considered as enough satisfactory to allow discussing the $\mu, \mathrm{B}, \mathrm{D}_{L D}, \mathrm{G}_{U D}$ parameters used in the simulation.

The frequency origins $\mathrm{G}_{L D}^{\text {droplet }}$ and $\mathrm{G}_{U D}^{\text {droplet }}$ are essentially determined by the mid distance between the $\mathrm{K}_{a}: 0 \leftarrow 1,1 \leftarrow 0$ bands. They differ by a blue shift of less than $0.2 \mathrm{~cm}^{-1}$ from those reported for the free complex when available. Such a small change of vibrational frequencies is general in HENDI spectroscopy. ${ }^{24}$ The response of the droplet is indeed much slower than vibrational frequencies. The small blue shift that is observed when a comparison with the free species is available likely reflects that for these specific vibrational excitations, the short range repulsive part of the acetylene-helium interaction has a larger effect than the long-range part. ${ }^{25}$

The separation between the $\mathrm{K}_{a}: 0 \leftarrow 1,1 \leftarrow 0$ bands is a stringent determination of the $\mathrm{B}$ constant whereas the large value of $\mathrm{D}$ serves to adjust the location of the $\mathrm{K}_{a}: 2 \leftarrow 1$ band, which otherwise would be located too far in the blue. The value of $\mu$ acts on the band shape. Its value is not very critical but it has to be significantly larger than the isolated complexes (15.75 u and $19.85 \mathrm{u}$ for $\mathrm{C}_{2} \mathrm{H}_{2}-\mathrm{Ar}$, $\mathrm{Kr}$, respectively). Otherwise the band widths would be too wide and too structured. The chosen values (33 $\mathrm{u}$ and $40 \mathrm{u}$, respectively) make the density of lines within the bands large enough to predict an adequate unstructured band profiles as observed experimentally. A $0.06 \mathrm{~cm}^{-1}$ linewidth is assumed in the simulation. It accounts for the excitation lifetime within the droplet, the laser width (1 MHz) being negligible at this scale. It falls in the same range as that observed by Nauta and Miller in their HENDI experiment on the $\mathrm{C}_{2} \mathrm{H}_{2}$ monomer. ${ }^{18}$

When calculating the bound states of the free $\mathrm{C}_{2} \mathrm{H}_{2}-\mathrm{RG}$ complexes, whatever the rare gas ( $\mathrm{Ne}, \mathrm{Ar}$ or $\mathrm{Kr}$ ), the rotational constant of $\mathrm{C}_{2} \mathrm{H}_{2}$ is taken to be that of the free $\mathrm{C}_{2} \mathrm{H}_{2}$ monomer $\left(B=1.17 \mathrm{~cm}^{-1}\right)$. In contrast, when the $\mathrm{C}_{2} \mathrm{H}_{2}-\mathrm{RG}$ complexes are embedded into the helium droplet, the effective rotational constant of $\mathrm{C}_{2} \mathrm{H}_{2}$ is not the same whether $\mathrm{C}_{2} \mathrm{H}_{2}-\mathrm{Ne}, \mathrm{C}_{2} \mathrm{H}_{2}-\mathrm{Ar}$ or $\mathrm{C}_{2} \mathrm{H}_{2}-\mathrm{Kr}$ is considered and in any case, it is not equal to that of the $\mathrm{C}_{2} \mathrm{H}_{2}$ monomer in the same environment. The effective value $\mathrm{B}=1.04 \mathrm{~cm}^{-1}$ of the $\mathrm{C}_{2} \mathrm{H}_{2}$ monomer becomes indeed $\mathrm{B}=1.14 \mathrm{~cm}^{-1}$ for $\mathrm{C}_{2} \mathrm{H}_{2}-\mathrm{Ne}$ and $\mathrm{B}=1.09 \mathrm{~cm}^{-1}$ for $\mathrm{C}_{2} \mathrm{H}_{2}-\mathrm{Ar}$ and $\mathrm{C}_{2} \mathrm{H}_{2}-\mathrm{Kr}$ (see Tab. 1). All these values are smaller than the rotational constant of free $\mathrm{C}_{2} \mathrm{H}_{2}\left(\mathrm{~B}=1.17 \mathrm{~cm}^{-1}\right)$. This is the manifestation that even in $\mathrm{C}_{2} \mathrm{H}_{2}-\mathrm{RG}$ complexes, the rotation of $\mathrm{C}_{2} \mathrm{H}_{2}$ is coupled to that of the non-superfluid component of the droplet, which "tries" to follow the molecular rotation. This is discussed in more detail below.

The decrease of $\mathrm{B}$ in the He-environment is significantly larger (11\% between 1.17 and $1.04 \mathrm{~cm}^{-1}$ ) for the $\mathrm{C}_{2} \mathrm{H}_{2}$ monomer than for $\mathrm{C}_{2} \mathrm{H}_{2}$ in the $\mathrm{C}_{2} \mathrm{H}_{2}-\mathrm{Ne}$ complex $(2.6 \%)$. This was interpreted in paper I as a repelling effect of the Ne-atom on the nonsuperfluid component of the droplet, which is therefore pushed away from the rotating $\mathrm{C}_{2} \mathrm{H}_{2}$ molecule. When $\mathrm{C}_{2} \mathrm{H}_{2}$ is bound to $\mathrm{Ar}$ or $\mathrm{Kr}$ instead of $\mathrm{Ne}$, the decrease of $\mathrm{B}$ goes up to $6.8 \%$. 
This indicates either a larger coupling of $\mathrm{C}_{2} \mathrm{H}_{2}$ with the nonsuperfluid component of the droplet or a larger extension of this component which increases its mass and momentum of inertia. Both effects are in line with the enhanced polarizability of the rare gas when $\mathrm{Ne}$ is replaced by $\mathrm{Ar}$ or $\mathrm{Kr}$. The He-Ar, $\mathrm{Kr}$ well depths $\left(2.09 \mathrm{meV}=16.9 \mathrm{~cm}^{-1}\right.$ and $2.13 \mathrm{meV}==17.2 \mathrm{~cm}^{-1}$, respectively) are both indeed 1.7 times larger than that of $\mathrm{He}-\mathrm{Ne}$ $\left(1.23 \mathrm{meV}=9.9 \mathrm{~cm}^{-1}\right) .{ }^{26}$ Apparently, this increased binding energy allows the Ar-atom and the $\mathrm{Kr}$-atom to compact the nonsuperfluid component of the droplet in the same way about $\mathrm{C}_{2} \mathrm{H}_{2}$. Nevertheless, this does not compensate totally the repelling effect due to the presence of a rare gas on one side of $\mathrm{C}_{2} \mathrm{H}_{2}$ since the effective value of $\mathrm{B}$ for $\mathrm{C}_{2} \mathrm{H}_{2}-\mathrm{Ar}, \mathrm{Kr}$ in the droplet $\left(1.09 \mathrm{~cm}^{-1}\right)$ is still larger than that of the embedded $\mathrm{C}_{2} \mathrm{H}_{2}$ monomer $\left(1.04 \mathrm{~cm}^{-1}\right)$.

The compaction effect of $\mathrm{Ar}$ and $\mathrm{Kr}$ on the non-superfluid component of the droplet has a counterpart on the dynamical parameter $\mu$. The apparent reduced mass of the $\mathrm{C}_{2} \mathrm{H}_{2}-\mathrm{Ar}$ (resp. $\mathrm{C}_{2} \mathrm{H}_{2}-\mathrm{Kr}$ ) pair switches indeed from $15.75 \mathrm{u}$ (resp. 19.85u) in the free complex to $33 \mathrm{u}$ (resp. $40 \mathrm{u}$ ) when the complex is in the helium environment. When the $\mathrm{C}_{2} \mathrm{H}_{2}-\mathrm{Ar}$, $\mathrm{Kr}$ complexes are coupled to the highly deformable non-superfluid component of the droplet, a few helium atoms are likely to be put into movement by the vibration along the van-der-Waals bond, hence increasing the reduced mass of the vibration partners. Such an effect is likely more pronounced with $\mathrm{Ar}$ or $\mathrm{Kr}$ than with Ne because of their larger polarizability. Actually, the increase of the apparent reduced mass is indeed a factor 2 with $\mathrm{C}_{2} \mathrm{H}_{2}-\mathrm{Ar}$ or $\mathrm{C}_{2} \mathrm{H}_{2}-\mathrm{Kr}$ and a factor 1.4 only with $\mathrm{C}_{2} \mathrm{H}_{2}-\mathrm{Ne}$ (see Tab. 1 ).

The increase of the reduced mass that has just been mentioned, a factor 2 for both $\mathrm{C}_{2} \mathrm{H}_{2}-\mathrm{Ar}$ and $\mathrm{C}_{2} \mathrm{H}_{2}-\mathrm{Kr}$ is a very large effect. It suggests that more almost $20 \mathrm{He}$ atoms could be driven by the vibration along the $\mathrm{C}_{2} \mathrm{H}_{2}-\mathrm{RG}$ van der Waals coordinate. Comparatively, the reduction of the rotational constant of $\mathrm{C}_{2} \mathrm{H}_{2}$ is not as large, a factor 1.07, whether the complex is free $\left(1.17 \mathrm{~cm}^{-1}\right)$ of embedded into helium $\left(1.09 \mathrm{~cm}^{-1}\right)$. This indicate a very different coupling efficiency of the non-superfluid component of the droplet with the two deformation coordinates of the complex. This suggests that, although helium atoms surround acetylene, they are mainly distributed about the triple bond of this molecule.

The effective constant $\mathrm{D}$ is also interesting to look at. We recall that its increase by four orders of magnitude when $\mathrm{C}_{2} \mathrm{H}_{2}$ is embedded in the droplet (from $1.6210^{-6}$ to $0.019 \mathrm{~cm}^{-1}$ ) does not reveal a drastic change of centrifugal corrections in the molecule. It appears as a fit parameter which helps accounting phenomenologically of an external perturbation, that due to the interaction between the molecular rotational state $j=2$ and the roton-maxon excitation of the superfluid component of the droplet. This was observed and discussed for the first time by Nauta and Miller. ${ }^{18}$ Later, this has been formalized and interpreted quantitatively by Zillich et al. in a a path-integral correlation function approach which confirms a strong coupling of the higher rotational states of the molecule with the roton and maxon excitations. ${ }^{27}$

The situation is comparable with the three $\mathrm{C}_{2} \mathrm{H}_{2}-\mathrm{RG}$ complexes listed in Tab. 1 since a four orders of magnitude increase of $\mathrm{D}$ is observed when these complexes are embedded into He-droplets. Again, the details are interesting. The value of $D$ is about the same for $\mathrm{C}_{2} \mathrm{H}_{2}$ and $\mathrm{C}_{2} \mathrm{H}_{2}-\mathrm{Ne}(0.019$ versus $0.0194 \mathrm{~cm}^{-1}$ ) whereas it is a factor 2.2 larger with $\mathrm{C}_{2} \mathrm{H}_{2}-\mathrm{Ar}$ and $\mathrm{C}_{2} \mathrm{H}_{2}-\mathrm{Kr}\left(0.042 \mathrm{~cm}^{-1}\right)$. This suggests that the coupling between the roton/maxon excitation of the helium superfluid component and the higher rotational states of $\mathrm{C}_{2} \mathrm{H}_{2}$ is much larger within the $\mathrm{C}_{2} \mathrm{H}_{2}-$ Ar complex than within $\mathrm{C}_{2} \mathrm{H}_{2}-\mathrm{Ne}$. This is a further demonstration of the larger well depths of the Ar-He and $\mathrm{Kr}$ He potentials, which compacts the helium environment about the complex.

\section{Summary and conclusion}

The dynamical effect of a helium droplet on the large amplitude deformations of the $\mathrm{C}_{2} \mathrm{H}_{2}-\mathrm{Ar}$, $\mathrm{Kr}$ complexes is explored in a joint experimental and theoretical work. Experimentally, a high resolution infrared spectroscopy work is performed, using the helium nanodroplet isolation technique (HENDI), in the vicinity of the $v_{3}, v_{2}+v_{4}+v_{5}$ Fermi dyad of acetylene.

Theoretically, spectra are simulated numerically. As in our former work on the $\mathrm{C}_{2} \mathrm{H}_{2}-\mathrm{Ne}$ complex, the dynamical effect of the helium environment is described phenomenologically by giving effective values to the reduced mass $\mu$ of the $\mathrm{C}_{2} \mathrm{H}_{2}-\mathrm{Ar}, \mathrm{Kr}$ pairs and to the constants $B$ and $D$ of the $\mathrm{C}_{2} \mathrm{H}_{2}$ molecule (rotational and centrifugal correction constants, respectively). The idea is to adjust these constants so the simulated spectrum fits with the experimental one.

Importantly, the assumption of our $\mathrm{C}_{2} \mathrm{H}_{2}-\mathrm{Ne}$ work that $\mathrm{C}_{2} \mathrm{H}_{2}$ is an almost free rotor within the complex is relaxed in the present work. This is justified here by calculations on the free $\mathrm{C}_{2} \mathrm{H}_{2}-\mathrm{Ne}$, Ar and $\mathrm{Kr}$ complexes.

As before with $\mathrm{C}_{2} \mathrm{H}_{2}-\mathrm{Ne}$, the adjusted values of $\mathrm{B}, \mathrm{D}$ and $\mu$ were used to document how the large amplitude motions of $\mathrm{C}_{2} \mathrm{H}_{2}$ within the $\mathrm{C}_{2} \mathrm{H}_{2}-\mathrm{RG}$ complex are perturbed by the helium environment. Qualitatively similar but quantitatively different effects are observed in the present $\mathrm{C}_{2} \mathrm{H}_{2}-\mathrm{Ar}, \mathrm{Kr}$ experiments compared with our former $\mathrm{C}_{2} \mathrm{H}_{2}-\mathrm{Ne}$ experiment. For instance, when comparing the values of $\mathrm{B}, \mathrm{D}$ and $\mu$ of the embedded $\mathrm{C}_{2} \mathrm{H}_{2}-\mathrm{Ar}, \mathrm{Kr}$ complexes to their counterpart in the free complexes, $\mathrm{B}$ decreases by $6.8 \%$ when $\mathrm{D}$ increases by four orders of magnitude larger and $\mu$ by a factor 2 . These observations are rationalized on the basis of a larger polarizability of Ar and $\mathrm{Kr}$ compared to Ne. The depth of the Ar-He and Kr-He potential are comparable and significantly larger than that of He-Ne. This results in the case of Ar and $\mathrm{Kr}$ (compared to $\mathrm{Ne}$ ) in an enhanced compaction effect of helium around the $\mathrm{C}_{2} \mathrm{H}_{2}-\mathrm{RG}$ complex which affects notably the coupling dynamics of the $\mathrm{C}_{2} \mathrm{H}_{2}-\mathrm{RG}$ large amplitude deformations with both the non-superfluid and the superfluid component of the helium environment.

\section{Conflicts of interest}

There are no conflicts to declare.

\section{Acknowledgement}

Financial support is acknowledged from: i) Agence nationale de la Recherche under grant ESBODYR ANR-14-CE06-0019; ii) CNRS GDR 3533 EMIE. E. Mengesha received support from Eu- 
rotalent under the 2015 project \#259.

\section{References}

1 S. Goyal, D. L. Schutt and G. Scoles, Phys. Rev. Lett., 1992, 69, 933-936.

2 J. P. Toennies and A. F. Vilesov, Annu. Rev. Phys. Chem., 1998, 49, 1-41.

3 J. P. Toennies, Mol. Phys., 2013, 111, 1879-1891.

4 Y. Kwon, P. Huang, M. V. Patel, D. Blume and K. B. Whaley, J. Chem. Phys., 2000, 113, 6469-6501.

5 M. Briant, E. Mengesha, P. de Pujo, M.-A. Gaveau, B. Soep, J.M. Mestdagh and L. Poisson, Phys. Chem. Chem. Phys., 2016, 18, 16414-16422.

6 J. Vanderauwera, D. Hurtmans, M. Carleer and M. Herman, J. Mol. Spectrosc., 1993, 157, 337-357.

7 C. R. Munteanu and B. Fernández, J. Chem. Phys., 2005, 123, 014309.

8 C. R. Munteanu and B. Fernández, J. Chem. Phys., 2016, 144, 119901.

9 C. Lauzin, E. Cauet, J. Demaison, M. Herman, H. Stoll and J. Lievin, Mol. Phys., 2012, 110, 2751-2760.

10 C. Lauzin, L. H. Coudert, M. Herman and J. Lievin, J. Phys. Chem. A, 2013, 117, 13767-13774.

11 M. Hartmann, F. Mielke, J. P. Toennies, A. F. Vilesov and G. Benedek, Phys. Rev. Lett., 1996, 76, 4560-4563.

12 R. Feltgen, H. Kirst, K. A. Kohler, H. Pauly and F. Torello, J. Chem. Phys., 1982, 76, 2360-2378.

13 O. Bunermann and F. Stienkemeier, Eur. Phys. J. D, 2011, 61, 645-655.

14 G. Guillon, A. Viel and J. Launay, J. Chem. Phys., 2012, 136, 174307-1-7.
15 A. Viel and J. Launay, J. Phys. Chem. A, 2014, 118, 65296535.

16 A. Simoni, A. Viel and J. Launay, J. Chem. Phys., 2017, 146, 244106.

17 P. F. Bernath, Spectra of Atoms and Molecules, 2nd ed, Oxford University Press, N.Y., 2005.

18 K. Nauta and R. E. Miller, J. Chem. Phys., 2001, 115, 8384 8392.

19 M. Briant, E. Mengesha, M. A. Gaveau, B. Soep, J. M. Mestdagh and L. Poisson, Phys. Chem. Chem. Phys., 2018, 20, 2597-2605

20 R. J. Bemish, L. Oudejans, R. E. Miller, R. Moszynski, T. G. A. Heijmen, T. Korona, P. E. S. Wormer and A. v. d. Avoird, J. Chem. Phys., 1998, 109, 8968-8979.

21 J. M. Hutson, in Advances in Molecular Vibrations and Collision Dynamics, ed. J. M. Bowman, JAI Press, 1991, vol. 1, pp. 146.

22 R. J. Bemish, P. A. Block, L. G. Pedersen, W. T. Yang and R. E. Miller, J. Chem. Phys., 1993, 99, 8585-8598.

23 S. Bratož and M. L. Martin, J. Chem. Phys., 1965, 42, 1051.

24 M. Y. Choi, G. E. Douberly, T. M. Falconer, W. K. Lewis, C. M. Lindsay, J. M. Merritt, P. L. Stiles and R. E. Miller, Int. Rev. Phys. Chem., 2006, 25, 15-75.

25 C. Callegari, A. Conjusteau, I. Reinhard, K. K. Lehmann and G. Scoles, J. Chem. Phys., 2000, 113, 10535-10550.

26 U. Buck, in Molecular Scattering; Physical and Chemical Applications, ed. K. P. Lawley, Wiley, Chichester,Sussex,England, 1975, pp. 313-388.

27 R. E. Zillich, Y. Kwon and K. B. Whaley, Phys. Rev. Lett., 2004, 93, 250401. 